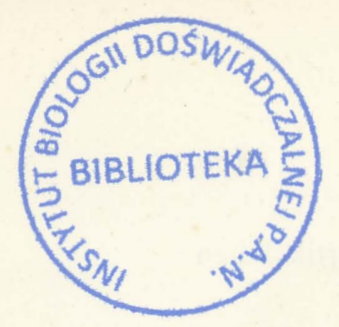

\title{
Neue Gattungen aus der Hymenopteren-Familie der Sphegiden.
}

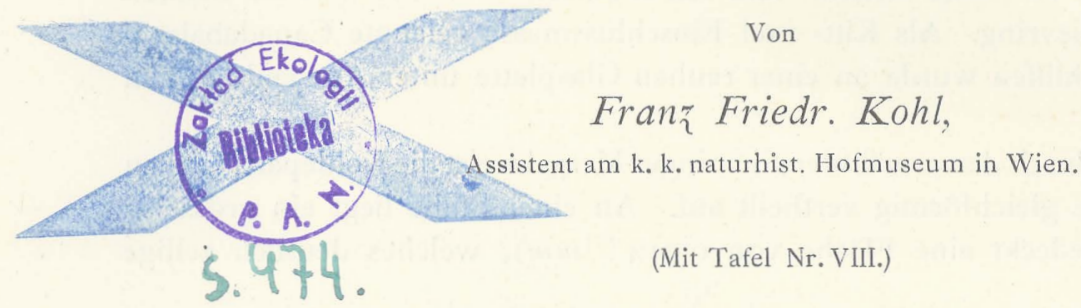

Durch die liebenswürdige Vermittlung des Herrn Custos Dr. Heinrich Dewitz wurde mir aus der königl. Sammlung in Berlin eine Anzahl Hymenopteren zur Ansicht anvertraut; darunter befanden sich zwei neue Gattungen von Grabwespen, von denen die eine zu der unter dem Namen "Larriden « bekannten Gattungsgruppe, die andere zur Gruppe von Philanthus gehört, welche in der nächsten Nähe der Larriden Platz nimmt. Da ich ferner in jüngster Zeit auch noch zwei andere neue Gattungsformen, die gleichfalls zu den Larriden gerechnet werden müssen, kennen gelernt habe, so schien es mir wünschenswerth, diese Gattungen im Interesse des systematischen Ausbaues zu untersuchen und zu beschreiben; hiebei wurde ich von dem Bestreben geleitet, diese Gattungen im System in möglichst natürlicher Anordnung — soweit es nämlich die lineare Anordnung der Formen in der Systematik überhaupt gestattet - einzureihen.

Zugleich ergreife ich diese günstige Gelegenheit, Herrn Dr. H. Dewitz, Custos am königl. Museum in Berlin, meinen herzlichen Dank auszudrücken für die Bereitwilligkeit, mit der er mich in meinen wissenschaftlichen Bestrebungen schon seit Jahren unterstützt.

\section{Sphodrotes nov. gen.}

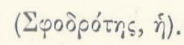

Oculi non renati mandibularum basim subtus attingunt $\left(\sigma^{7}\right)$. Orbita interiora parallela. Stemmata normalia. Palpi maxillares 6 -articulati, labiales 4 -articulati. Mandibulae interdum margine exteriore profunde excisae. Antennae filiformes i 3 -articulatae $\left(\sigma^{7}\right)$.

Collare discretum pronoto vix humilius. Sutura episternalis mesopleurarum discreta, Segmentum medianum postice fere ad perpendiculum abscissum, lateribus marginatum. Segmentum ventrale secundum (segmenti $3^{\text {tii }}$ ) valde convexum.

Pedes spinulosi. Tibiae intermediae I-calcaratae. Coxae intermediae non contiguae, sed parum tantum distant. Unguiculi pulvillis validis.

Alae anteriores (Tab. VIII, Fig. I3) magnae, areola radiali apice subrotundata, appendiculata; areola cubitalis prima quam secunda petiolata et tertia subrhomboidea simul sumptis fere major; tertia venam recurrentem primam et secundam excipit. Vena basalis alae anterioris extra areolam submedialem primam egrediens. - Areola submedialis alae posterioris post originem venae cubitalis terminata.

Corpus nigro-atrum, absque splendore ullo metallico, grosse punctatum. Feminae adhuc incognitae. 
Sphodrotes, aus Neu-Süd-Wales stammend, zeigt sehr grosse Verwandtschaft zu mehreren Gattungen, welche unanfechtbar in die Nähe der Gattungsgruppe der gemeinen Larriden gestellt werden müssen und in unverkennbarer Weise die Verbindung dieser mit den Artgruppen von Nysson und wohl auch anderen Nyssoniden (Alyson, Didineis) herstellen. Diese Gattungen sind Tachyrhostus Sauss., Helioryctes Smith (Cat. Hym. Brit. Mus., P. IV., I 856), Bothynostethus Kohl und Scapheutes Handlirsch.

Von Tachyrhostus unterscheidet sich Sphodrotes: 1. durch die abgestutzte, verhältnissmässig kurze, übrigens ebenfalls mit der Anlage einer »Anhangszelle v versehene Radialzelle, 2. die gestielte Form der zweiten Cubitalzelle, 3. die Art des Verlaufes der Discoidalqueradern - es nimmt nämlich die zweite Cubitalzelle beide auf, während bei Tachyrhostus die erste deutlich und beständig in die erste, die zweite in die zweite Cubitalzelle mündet -4 . durch den Ursprung der Basalader, welcher kein interstitialer ist, sondern sehr entschieden nach dem Abschlusse der ersten Submedialzelle, also an der zweiten erfolgt, 5. die grobe Punktirung des Körpers.

Uebrigens ist die Verschiedenheit des Kopfes, der, weil dicker, nicht so sehr linsenförmig ist, die viel bedeutendere Länge der sich nirgends verdickenden Fühler, das längere, mit keiner Mittelrinne auf der Rückenfläche ausgezeichnete Mittelsegment für die Kennzeichnung von Sphodrotes nicht werthlos.

Von der Gattung Helioryctes Smith, die mir, beiläufig bemerkt, nur aus der Beschreibung und der dieser beigegebenen Abbildung bekannt ist, unterscheidet sich Sphodrotes: I. durch den Verlauf der ersten Discoidalquerader, der bei ersterem in der Weise wie bei Tachyrhostus erfolgt, 2. die Gestalt des Kopfes, die Länge und Gestalt der Fühler, besonders die Länge des Mittelsegmentes, 3. die wie bei Nysson-Arten mehr gewölbte Form des zweiten Hinterleibssegmentes - bei Helioryctes erscheint dieses vorne fast wie abgeschnitten und zeigt nur eine sehr schmale Rückenfläche 4. durch die grobe Punktirung des Körpers. Vielleicht würde die Autopsie der Smith'schen Gattung auch noch andere Gattungsunterschiede ergeben.

Vergleicht man ferner Sphodrotes mit Scapheutes, so ergibt sich ein Unterschied in der Art des Ursprunges der Basalader der Vorderflügel und Cubitalader der Hinterflügel - bei letzterer entspringt nämlich sowohl die Basalader der Vorderflügel als auch die Cubitalader der Hinterflügel noch an der ersten Submedialzelle des bezüglichen Flügels - ferner in der Gesichtsbildung, vorzüglich bedingt durch die bei Scapheutes gegen den Kopfschild convergenten inneren Augenränder.

Von Bothynostethus unterscheidet sich unsere Gattung: 1. durch die nicht convergenten inneren Augenränder, 2. die Anlage der Anhangszelle, 3. die gewöhnliche Gestalt der Hinterschenkel - bei Bothynostethus nehmen sie gegen das Ende an Breite stets zu - 4. die sichtlich bedornten Schienen, 5. die Punktirung des Körpers.

Schliesslich ist Sphodrotes noch mit Nysson zu vergleichen, von welcher Gattung sie sich I. durch die Anlage einer Anhangszelle, 2. die Art des Ursprunges der Basalader der Vorderflügel, 3. durch die einspornigen Mittelschienen und 4. die parallelen, nicht gegen den Kopfschild zusammenneigenden inneren Augenränder unterscheidet.

Sphodrotes punctuosa n. sp.

Länge $8 \mathrm{~mm}$. ơ. Mattschwarz. Kniee, Schienen, Tarsen und zum Theile auch die Fühler braunroth. Hinterränder der Hinterleibsringe und Gesicht mit gelblicher Pubescenz besetzt. Flügel brïunlich getrübt. Kopfform: Taf. VIII, Fig. I.

Oberkiefer am Innenrande, nahe bei der Basis mit zwei unbedeutenden Zähnchen. Kopfschild flach, mit bogig verlaufendem Vorderrande. Stirne etwas eingedrückt; in dem flachen Eindrucke, der beiderseits nahe den inneren Augenrändern und parallel mit diesen 
eine Art Leisten bildet, bewegen sich die Fühlerschäfte. Fühler nirgends verdickt. Schaft fast so lang wie das zweite und dritte Geisselglied. - Erstes Geisselglied kugelig, zweites etwa doppelt so lang als am Ende dick, die folgenden (drittes, viertes und fünftes) ein wenig kürzer. Hintere Nebenaugen von einander fast ebensoweit abstehend wie von den Netzaugen. Schläfen kräftig. Der Hinterkopf (Taf. VIII, Fig. 10) verschmälert sich nach hinten und ist sehr scharf gerandet. Kopf sehr grob und dicht punktirt.

Der Thorax ist gleichfalls sehr grob und dicht punktirt. Collare gut entwickelt, nicht unter die Fläche des Dorsulum herabgedrückt.

Mittelsegment breiter als lang, oben grob netzartig gerunzelt, die hintere, abstürzende Fläche ist gleichfalls grob und fast netzartig gerunzelt und an den Seiten gerandet; die Mittelsegmentseiten sind mit groben Längsstreifen versehen. Das zweite Hinterleibssegment ähnelt in seiner Gestalt dem von Ny-sson ommissus, ebenso das dritte oben und unten. Die Hinterleibsringe $2-8$ sind wie der Thorax grob und ziemlich dicht punktirt. Die Punkte glänzen am Grunde, Beine kräftig, auch die Tarsen. Der längere Schienensporn der Hinterbeine überragt die Hälfte des Metatarsus, der reichlich so lang ist wie die zwei folgenden Glieder.

Neu-Süd-Wales.

\section{Laphyragogus nov. gen.}

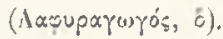

Oculi renati subtus mandibularum basim attingunt $(q)$.

Stemmata normalia. Mandibulae externe prope medium excisae. Palpi maxillares 6-articulati, labiales 4-articulati. Antennae setaceae, 12 -articulatae in femina.

Collare vix discretum pronoto multo humilius. Sutura episternalis mesopleurarum discreta. Segmentum medianum postice ad perpendiculum abscissum, tibiae tarsique ciliata. Metartasus pedum posteriorum processu lobiformi instructus. Tibiae intermediae I-Calcaratae. Coxae validae, intermediae contiguae. Unguiculi pulvillis carent.

Alae (Tab. VIII, Fig. 2 r) magnae, areola radiali lanceolata, oblique truncata, areolis cubitalibus tribus magnitudine fere aequalibus, secunda venam recurrentem primam et secundam excipiente. Vena basalis extra areolam submedialem primam egrediens.

Areola submedialis alae inferioris post originen venae cubitalis terminata.

Corpus ubre flavopictum. - Mares adhuc incogniti.

Was die Stellung der Gattung Laphyragogus im System betrifft, so steht sie in Bezug auf die Beschaffenheit des Flügelgeäders am nächsten der Gattung Astatus Latr. Die Ausrandung der Augen, die Bildung des Hinterleibes, besonders die der oberen Afterklappe, und die reiche Zeichnung deuten auf Beziehungen zu Philanthus hin, während die geringe Entwicklung des Collare, der Ausschnitt am Aussenrande der Oberkiefer, die Bildung des Mittelsegmentes und der Besitz einer Anhangszelle in den Vorderflügeln auf eine nähere Verwandtschaft mit so manchen gemeinen LarridenGattungen (Tachysphex, Tachytes, Notogonia, Dinetus) schliessen lässt; mit letztgenannter Art hat er, abgesehen von sehr grosser Flügelverwandtschaft und der Regelmässigkeit der hinteren Nebenaugen, auch die reiche Körperzeichnung gemein.

Nach diesen Umständen scheint es mir völlig gewiss, dass Laphyragogus am besten wird neben Dinetus gestellt werden können, als ein Glied, welches zu Philanthus (sens. lat.) führt.

Laphyragogus pictus n. sp. $\$$.

Länge $8-9 \mathrm{~mm}$. Schwarz. Hinterleib, abgesehen von den blassgelben Binden, orangeroth, zum Theile ins Schwarze übergehend. Körper sehr reichlich gezeichnet, 
jedoch ohne Zweifel in veränderlicher Weise. Blassgelb sind: der Kopfschild zum Theile, das Pronotum stellenweise, die Schulterbeulen, eine Makel unmittelbar dahinter, die Flügelschuppen, der Hinter- und Seitenrand des Schildchens, das Hinterschildchen, zwei sehr grosse Seitenmakeln aur dem Mittelsegmente, breite Binden auf den übrigen Hinterleibsringen - die beiden Endringe sind oben ganz gelb, die Schienen und Tarsen, zum Theile auch die Schenkel, die überdies noch lehmgelbe Stellen tragen, und endlich das Geäder der wasserhellen Flügel. - Pubescenz weiss.

Oberkiefer mit einer Spitze, am Aussenrande nahe bei der Mitte (der Basis näher als der Spitze) ausgeschnitten. Kopfschild ziemlich flach, sein Vorderrand verläuft im Bogen. Ausrandung der Augen nicht stark, auf dem Scheitel nähern sie sich bis auf die Länge des zweiten, dritten und vierten Geisselgliedes. Hintere Nebenaugen von einander reichlich so weit abstehend wie von den Netzaugen. Hinter den Augen erscheint der Scheitel gar nicht fortgesetzt; auch die Schläfen sind ungemein schmächtig. Auffallend gross ist der trapezförmig gerandete Ausschnitt des Hinterhauptes. (Taf.VIII, Fig. 6 und 7.)

Die Fühler stehen am Grunde etwa um die Länge des dritten Geisselgliedes von einander ab. Der Fühlerschaft ist kurz, kürzer sogar wie das zweite Geisselglied. Erstes Geisselglied rundlich, zweites $2 \cdot 5 \mathrm{mal}$ so lang wie dick, drittes, viertes, fünftes, sechstes kaum doppelt so lang wie dick, walzig. Stirne und Scheitel deutlich und ziemlich dicht punktirt, erstere nur schwach gewölbt.

Collare sehr tiefliegend, unscheinbar. Dorsulum glänzend, punktirt, in der Mittelpartie nur höchst sparsam, bei den Rändern dichter. Schildchen flach, polirt glänzend, mit einzelnen Punkten. Mesopleuren Iederartig, runzelig, matt. Metapleuren vertieft.

Mittelsegment breiter wie lang, hinten senkrecht abstïrzend, auf der Rückenfläche netzartig, im Uebrigen mehr lederartig gerunzelt. Hinterleib sonst äusserst fein und dichtmaschig gerunzelt, ziemlich flach, obere Afterklappe flach, dreieckig.

Hüften sehr kräftig. Schenkel schwach, die vordersten am Unterrande in einer Linie, ferner sämmtliche Schienen allenthalben bewimpert. Der vorderste Tarsus (Taf. VIII, Fig. 9) jst höchst merkwürdig gebildet: der Metatarsus würde nicht länger erscheinen als das nächstfolgende Glied, wenn er nicht an der Aussenseite, also einseitig in einen langen, lappenförmigen Fortsatz ausgezogen wäre; auch das zweite, dritte und vierte Tarsenglied sind an der Aussenseite ausgezogen, unsymmetrisch. Ausserdem tragen die vordersten Tarsen an der Aussenkante einen langen Wimpernkamm. Die Mittel- und Hintertarsen sind rings bewimpert. Der längere Sporn des hintersten Beinpaares hat ungefähr die Länge des Metatarsus, der etwa so lang ist als die drei folgenden Glieder zusammengenommen.

Flügelgeäder: Taf. VIII, Fig. 2 I. Frenalhäkchen zähle ich an der Type 13, die in ununterbrochener Reihenfolge angeordnet sind.

Die Type stammt aus Egypten und befindet sich nunmehr im Besitze des königl. zool. Museums in Berlin.

\section{Homogambrus nov. gen.}

$$
\text { (Ouorzu. } \beta_{100}, \dot{0} \text { ) }
$$

Oculi non renati maris ut in genere Astato superne connati subtus mandibularum basim attingunt. Ocelli posteriores obsoletissimi, difformes, oblongi. Mandibulae breves externe prope medium excisae. Palpi maxillares 6-articulati, labiales 4 -articulati. Antennae setaceae.

Collare vix discretum, pronoto muito humilius. Sutura episternalis mesopleurorum bene discreta. Mesosternum utrinque tuberculo armatum. 
ich hätte ihn für identisch angesehen, wenn nicht an den Metapleuren die Episternalnaht fehlte. Zudem entspringt übereinstimmend mit Anthophilus die Cubitalader der Hinterflügel an der Submedialzelle noch vor ihrem Abschlusse, während sie bei Aphilanthops in sichtlicher Entfernung von der Medialader abgeht.

Es handelt sich nun um die Frage, ob man ihn als Gattung hinstellen oder nur als cine natürliche Artgruppe von Philanthus auffassen soll. Bei den engen Beziehungen von Philanthus (sens. str.), Anthophilus, Trachypus, Aphilanthops und Philoponus, die alle besonders durch die Beschaffenheit der Gesichtsbildung, die Bartfransen der Seiten des Kopfschildrandes, die Anlage des Flügelge:̈ders, die Bewehrung der Beine und die habituelle Aehnlichkeit ausgesprochen erscheinen, glaube ich, dass es am natürlichsten wäre, alle diese Gattungen unter dem Namen Philanthus (sens. lat.) als natürliche Gattung zusammenzufassen; die übrigen Bezeichnungen mögen immerhin für die Gruppirung der Arten noch fortbestehen. Die geringste Existenzberechtigung hätte, beiläufig bemerkt, $A n$ thophilus, der allein nur auf die Ursprungsart der Cubitalader der Hinterflügel gegründet ist.

Philanthus (sens. lat.) würde dann in einer Weise aufgefasst, die analog ist mit der Auffassung der Gattungen Crabro (Mor. Thoms. Kohl), Gorytes im Sinne Handlirsch's, Stizus (Handl.), Sphex (Kohl), Ammophila (Kohl), Pompilus (Kohl), Salius (Kohl), Mutilla (Gerst.) u. a.

\section{Uebersicht der natürlichen Gattungsgruppen von Philanthus.}

I. Netzaugen am Innenrande ausgerandet, nierenförmig (manchmal nur in schwachem Grade). [Schulterbeulen bis zu den Flügelschuppen hinaufreichend. Randbärte der Kopfschildseiten gut ausgeprägt].

- Netzaugen am Innenrande nicht ausgerandet. [Randbärte der Kopfschildseiten weniger deutlich, wenngleich vorhanden. Augen die Oberkiefer - so weit bekannt - im männlichen Geschlechte nicht vollständig erreichend]. . . .

2. Zweiter Hinterleibsring stielförmig gestreckt. [Augen die Oberkiefer auch im männlichen Geschlechte erreichend. Die Cubitalader der Hinterfügel entspringt interstitial im Abschlusse der ersten Submedialzelle oder aber noch an dieser vor dem Abschlusse. - Neotropische Region.] Trachypus-Gruppe (Klug.).

- Zweiter Hinterleibsstiel nicht stielförmig, wenngleich manchmal etwas verschmälert und abgeschnürt. Augen die Oberkiefer im männlichen Geschlechte bei den meisten Arten nicht erreichend . . . . . . . . . . . . .

3. Die Cubitalader der Hinterflügel entspringt nach dem Abschlusse der ersten Submedialzelle an der Medialader. [Scheint nur der Austral-Region zu fehlen.]

Philanthus-Gruppe (genuin).

-- Die Cubitalader der Hinterflügel entspringt vor dem Abschlusse der Submedialzelle, also noch an dieser. [Scheint nur der Austral-Region zu fehlen.]

Philanthus-Gruppe (Anthophilus). (Scheint nur künstlich zu sein.)

4. Episternalnaht der Mesopleuren vorhanden. Schulterbeulen nicht vollkommen bis zu den Flügelschuppen hinaufreichend. Die Cubitalader der Hinterflügel entspringt sehr entschieden nach dem Abschlusse der ersten Submedialzelle, also nicht mehr an dieser, an der Medialader. [Nearktische Region.]

Aphilanthops-Gruppe (Patton.).

-- Episternalnaht der Mesopleuren fehlend. Schulterbeulen bis zu den Flügelschuppen hinaufreichend. Die Cubitalader der Hinterflügel entspringt vor dem Abschlusse der ersten Submedialzelle, also noch an dieser. [Mediterran-Region.]

Philoponus-Gruppe (Kohl). 
Oberkiefer spitz, am Innenrande mit einem Zähnchen bewehrt, am Aussenrande bei der Mitte tief ausgeschnitten. Mitteltheil des Kopfschildes mit deutlich abgesetztem Vorderrande; dieser erscheint, weil mit zwei sehr seichten Ausrandungen versehen, unvollkommen dreizähnig. Stirne und Kopfschild mit silberweiss glänzender Pubescenz bekleidet. Stirne stark gewölbt, in der Mitte, d. i. ungefähr in einem Abstande vom Fühlergrunde, welcher der Länge des Fühlerschaftes gleichkommt. Hintere Nebenaugen sehr undeutlich, gestreckt und flach, eingezwängt zwischen den Netzaugen und einem kreisrunden Höcker, vor dem knapp das vordere regelmässige Nebenauge sitzt, hinten aneinander stossend. Fühlerschaft am Grunde und an der Spitze gelblich, ungefähr so lang wie die drei ersten Geisselglieder zusammen. Erstes Geisselglied so lang wie breit, zweites reichlich doppelt so lang als am Ende dick, drittes, viertes und fünftes unbedeutend kürzer, etwa doppelt so lang wie dick.

Bruststück glänzend. Dorsulum im vordersten Dritttheil sehr fein und ziemlich dicht punktirt, die Punkte etwas undeutlich; ähnlich punktirt sind auch die Mesopleuren stellenweise. Mittelsegment breiter als lang, auf dem Rücken durch eine erhabene Längslinie halbirt, beiderseits von dieser schräg gestreift. Die Streifen greifen auch ein wenig auf die Mittelsegmentseiten über, die im Uebrigen glänzend glatt sind. Die senkrecht abstürzende hintere Fläche des Mittelsegmentes ist quergestreift und wie bei T'achysphexArten mit einer Grube ausgestattet.

Hinterleibsringe oben dicht und äusserst fein punktirt, mit schwacher Pubescenz besetzt. Die Bauchringe sind spärlich punktirt, glänzend. Der fünfte und sechste Bauchring zeigt erhabene quergestellte Seitenleisten, die ungefähr in der Mitte der Länge der Ringe sitzen; sie ragen gegen die Seiten hin stärker empor als gegen die Mitte der Breite.

Die Flügel (Taf. VIII, Fig. I 6) sind wasserhell, nur in der Cubital- und Discoidalzelle leicht bräunlich getrübt.

Nach den nahen Beziehungen zu Piagetia kann Homogambrus nur bei der Gattungsgruppe der genuinen Larriden, am besten wohl zwischen Piagetia und Lyroda untergebracht werden.

\section{Philoponus}

(neue natürliche Artgruppe der Gattung Philanthus sens. lat.)

(Dı).órovos, о.)

Oculi non renati mandibularum basim subtus fere attingunt. Orbita interiora fere parallela verticem versus vix convergunt. Stemmata normalia.

Palpi maxillares 6-articulati, labiales 4-articulati. Mandibulae in margine exteriore non excisae, integrae. Antennae breviusculae. Collare pronoto non humilius.

Mesopleurae sutura e pisternali carent. Tubercula humeralia alarum tegulas ut in genere Philantho attingunt. Segmentum secundum abdominis nonnihil constrictum.

Coxae intermediae distant. Unguiculi pulvillis validis. Tibiae intermediae $\mathrm{r}$-calcaratae. Pedes spinulosi.

Alae anteriores area radiali lanceolata, acuminata; areolis cubitalibus tribus, quorum prima secundae una cum tertia magnitudine aequalis est; secunda trapezina quan tertia trapezoidea paullo minor. Secunda excipit venam recurrentem primam, tertia secundam. Vena basalis interstitialis.

Areola submedialis alae inferioris post originem venae cubitalis terminata.

Corpus ubre flavido-pictum. - Feminae adhuc incognitae.

Philoponus steht der im Jahre i 88I von Patton (Proceed. Boston Soc. of Nat. Hist. XX, p. 403) beschriebenen nordamerikanischen Gattung Aphilanthops sehr nahe; 
Segmenta ventralia 5 et 6 utrinque cristae instar transverse elevata.

Tibiae intermediae I-calcaratae. Unguiculi pulvillis distinctis instructi. Coxae intermediae distantes.

Alae superiores magnae abdomen paullo superantes, area radiali brevi late truncata (appendiculata) areolis cubitalibus tribus, secunda trapezina venam recurrentem primam et secundam excipiente, vena basali postcostam a stigma longe remote attingente et longe extra areolam submedialem primam egrediente. Alae inferioris vena cubitalis extra aream submedialem oritur.

Feminae adhuc incognitac.

Ferd. Morawitz beschreibt einen Tachysphex (?) globiceps, von dem er selbst glaubt, dass er einer anderen Larriden-Gattung angehören dürfte. Das k. k. Hofmuseum erwarb vor Kurzem eine Sammlung von Hymenopteren aus Russisch-Armenien (Araxesthal), in welcher sich zwei mit der Morawitz'schen Beschreibung des Tachysphex globiceps vollkommen übereinstimmende Thiere befanden.

Es wurde mir sogleich klar, dass Tachysphex globiceps zu einer anderen Gattung gestellt werden muss, da er von Tachysphex nicht nur durch die ganz wie bei AstatusMännchen ${ }^{1}$ ) am Scheitel zusammenstossenden Netzaugen, sondern auch durch den Mangel einer Ausrandung in der Nähe des Schenkelgrundes, die Beschaffenheit der hinteren Bauchringe, sowie die so sehr breit abgestutzte, kurze Radialzelle verschieden ist.

Das Flügelgeäder (Taf. VIII, Fig. I6) stimmt mit dem von Piagetia vollständig überein. Ich wäre beinahe geneigt gewesen, den Tachysphex globiceps für das Männchen einer Piagetia-Art, und zwar für das der Piagetia mongolica F. Mor. (Hor. Soc. Ent. Rossi XXIII, I889, p. I 30) zu halten, allein nach den Beschreibungen der PiagetiaMännchen (PiagetiaWoerdeni und Ritsemae) stossen die Augen auf dem Scheitel nicht zusammen und sind die Hinterschenkel mit einem zahnartigen Fortsatz bewehrt; auch erscheinen die Bauchringe ohne Erhabenheiten und das zweite Segment des Hinterleibes verlängert und verschmächtigt.

Da die Zeitschrift der Hor. Soc. Ent. Rossi vielen Hymenopterologen nicht zugänglich ist, mag eine nochmalige Artbeschreibung, die zum Theil ergänzend ist, gut am Platze sein.

Länge $6-7 \mathrm{~mm}$. $\mathrm{o}^{7}$. Schwarz. Hinterleib zum Theile roth (am zweiten und dritten Ringe). Blassgelb gefärbt sind die Taster, Oberkiefer (zum Theile), Kniee, Schienen und Tarsen, sowie die ganze Bedornung.

1) Unter den erwähnten Hymenopteren aus dem Araxesthal befand sich auch eine prächtige neue Astatur-Art, die ich Astatus scapularis (q) nenne. Sie hat die Grösse eines kleineren Stückes von Astatus boops Schr., der Hauptsache nach auch deren Färbung. Roth sind das zweite und dritte Segment, häufig auch das vierte mehr weniger. Schulterbeulen elfenbeinweiss. Weisse Haare zeigen sich nur auf den Schläfen und sehr spärlich auf dem Mittelsegmente. Die Beborstung des Bruststückes ist kurz, braunschwarz. Mittleres Kopfschiidplättchen etwas aufgehoben und in Folge eines winkeligen Ausschnittes zweizipfelig, nicht wie bei boops abgestutzt. Geringster Abstand der Netzaugen auf dem Scheitel geringer als bei boops; er beträgt nur die Länge des ersten und zweiten Geisselgliedes, nicht wie bei diesem die des zweiten und dritten. Die Fühlerglieder zeigen keinen erheblichen. Unterschied bein Vergleiche mit denen von boops. Stirne, Scheitel, Dorsulum und Schildchen mit sehr groben Punkten; auf der Stirne und dem Vorderdritttheil des Dorsulums stehen sie in mässiger Dichte, im Cebrigen mehr zerstreut. Die Punkte auf dem Dorsulum und Schildchen sind tief gestochen, ebenso die auf den Mesopleuren; diese erscheinen glänzend, da zwischen den Punkten deutliche glatte Zwischenflächen Platz finden. Mittelsegment zerknittert gerunzelt, ähnlich wie bei boops, nur etwas dichter. Die obere Afterklappe erscheint anders geformt, schmäler. (Taf. VIII, Fig. I8.) Flügel wie bei boops. Auch aus Sarepta befindet sich ein Weibchen dieser schönen Art in der Sammlung des k. k. naturhist. Hofmuseums . 
NB. Bei der Gruppirung der Philanthus-Arten wäre noch die Gattung Nectanebus Spinola (Ann. soc. entom. de France, I 838, 489) zu berücksichtigen, die ich nicht kenne. Nach der Beschreibung scheint sie dem Philoponus nahe zu stehen. Bei ihr ist jedoch die Radialzelle abgerundet. Wie es mit der Episternalnaht, den Schulterbeulen etc. steht, können wir aus der Beschreibung leider nicht ersehen.

Philoponus Dewitzii n. sp.

Länge $6 \mathrm{~mm}$. $\sigma^{7}$. Schwarz. Kopfschild und Oberkiefer elfenbeinfarben. Fühler lehmfarben. Gelblichweiss sind: der obere Theil des tief ausgerandeten Collare, die Schulterbeulen und Flügelschuppen, manchmal Makeln auf dem Schildchen, das Hinterschildchen, die Binden auf den Hinterrändern der Rückensegmente, die Schienen und Tarsen, theilweise auch die Schenkel. Flügel ziemlich hell. Pubescenz weiss.

Oberkiefer ohne Ausschnitt, einspitzig. Kopfschildmitteltheil von sehr leichter Wölbung, sein Vorderrand mit vier stumpfen Zähnchen bewehrt. Gesicht mit silberweisser Pubescenz. Nebenaugen von einander so weit abstehend wie von den Netzaugen. Diese erreichen die Oberkiefer nicht ganz. Scheitel und Schläfen glänzend undeutlich punktirt. Fühler unregelmässig. (Taf. VIII, Fig. 5.) Schaft kurz. Drittes Geisselglied ebenfalls sehr kurz, kaum länger als das vierte, fünfte, sechste und siebente, kürzer als die beiden Endglieder; das letzte endigt stumpf, erscheint gebogen, das zehnte, eilfte und zwölfte Fühlerglied erscheinen an der Unterseite am Ende ausgezogen, so dass daselbst der Fühler fast gesïgt aussieht. (Taf. VIII, Fig. 5.)

Das Collare steht im Ganzen in gleicher Höhe wie das Dorsulum, vorne fällt es senkrecht ab; merkwürdig ist die tiefe Ausrandung. (Taf. VIII, Fig. 26.) Mesothorax glänzend; Mesopleuren undeutlich punktirt. Das Mittelsegment ist in ähnlicher Weise wie bei echten Philanthus-Arten gebildet, zum Theile fein runzelstreifig, mit einer glatteren, eine Art »herzförmigen Raumes « umgrenzenden Stelle. Das zweite Hinterleibssegment erscheint vom nächsten deutlich abgeschnürt; es ist viel kürzer als breit. Die Rückensegmente sind glänzend, sparsam punktirt.

Bedornung der Beine wie bei echten Philanthus-Arten. Metatarsus der Mittelbeine ungefähr von der Länge der beiden folgenden Glieder, der der hinteren etwas länger.

Die Art ist Herrn Custos Dr. Heinrich Dewitz in Berlin zubenannt.

$\mathrm{Zu}$ Philoponus gehört auch Anthophilus variegatus Ferd. Morawitz. Der geschätzte Autor hat im Tauschwege ein Stück an unser Museum abgegeben; es ist das Stïck, von welchem er in der Beschreibung erwähnt, dass es auf der Kopfschildscheibe mit einer weissen Makel gezeichnet ist. Dieses Stück ist jedoch ein Männchen. In der Gestalt des Collare unterscheidet es sich auffallend von Philoponts Dewitzii; dieses ist nämlich nicht ausgerandet und im Vergleiche mit echten Arten von Philanthus sehr stark.

Philoponus variegatus hat keine Kopfschildzähne, der Mitteltheil des Kopfschildes erscheint quer abgestutzt. Fühler gewöhnlich. in Berlin.

Egypten. Die Typen befinden sich in der Sammlung des königl. zool. Museums

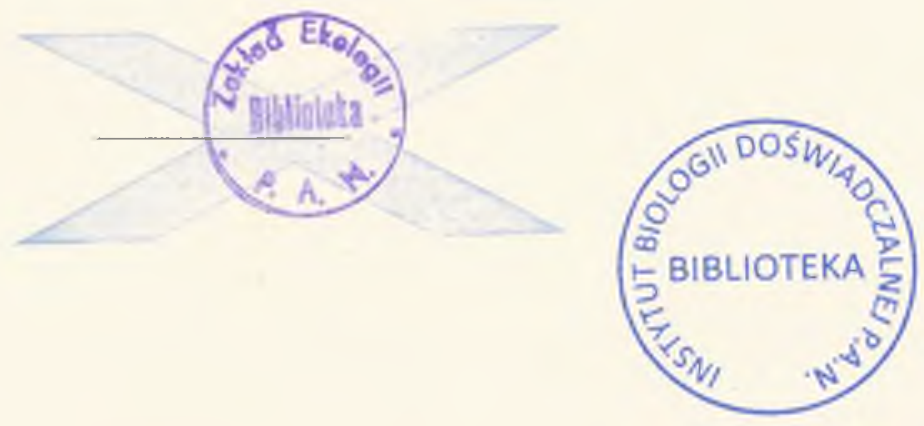




\section{Erklärung zu Tafel Nr. VIII.}

I. Kopf von Sphodrotes n. g.

2. Fühler von Laphyragogus pictus Kohl $\sigma^{\circ}$.

3. Fühler von Astatus scapularis Kohl $q$.

4. Kopf von Homogambrus n. g.

j. Fühler von Philanthus (Philoponus) Dewitzii Kohl ot.

6. Kopf von Laphyragogus pictus Kohl \& (von oben).

7. Kopf von Laphyragogus pictus Kohl $\bigcirc$ (von hinten).

8. Mittelbein von Laphyragogus pictus Kohl $\bigcirc$.

9. Vorderbein von Laphyragogus pictus Kohl $q$.

Io. Kopf von Sphodrotes punctuosa Kohl ơ (von oben).

11. Kopf von Laphyragogus pictus kohl + .

12. Kopf ron Philanthus (Philoponus) Dewitzii Kohl $\sigma^{x}$.

13. Flügelgeäder von Sphodrotes punctuosa KohI ơ'.

14. Kopf von Tachyrhostus relucens Smitl.
15. Kopf von Philoponus Devvitz̧ii Koh1 ơ (von oben).

I6. Flügelgeäder von Homogambrus n. g.

17. Hinterbein von Philoponus Dewit 7 ii Kohl ơ

18. Pygidium von Astatus scapularis Kohl $\&$.

19. Pygidium von Homogambrus n. g.

20. Mittelbein von Philanthus (Philoponus) Dewit Tii Kohl $\sigma^{x}$.

2 I. Flügelgeäder von Laphyragogus pictus Kohl ơ 22. Pygidium von Aphilantops Patton.

23. Hinterleibsstiel von Philanthus aus der Gruppe Trachypus.

24. Hinterleib von Sphodrotes punctuosa Kohl o (Seitenansicht).

25. Flügelgeäder von Philanthus (Philoponus) Dewit ii Kohl $\sigma^{7}$.

26. Collare von Philanthus (Philoponus) Dewitzii Kohl $O^{7}$ (von vorne gesehen).

27. Pygidium von Astatus boops Schrank 9 . 
Kohl: Neue Gattumgenvon Sphegiden.

Taf.VIII.
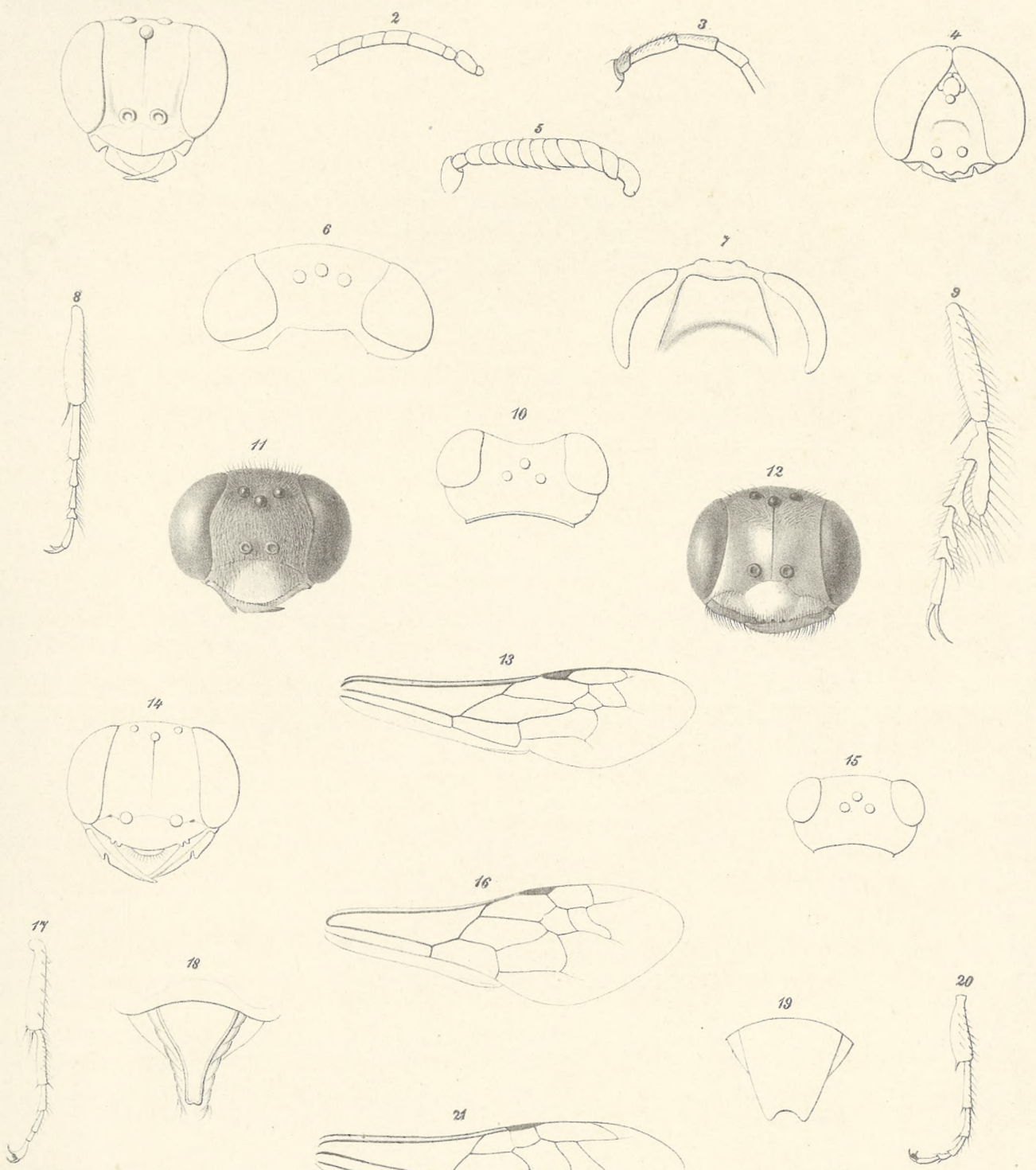

22
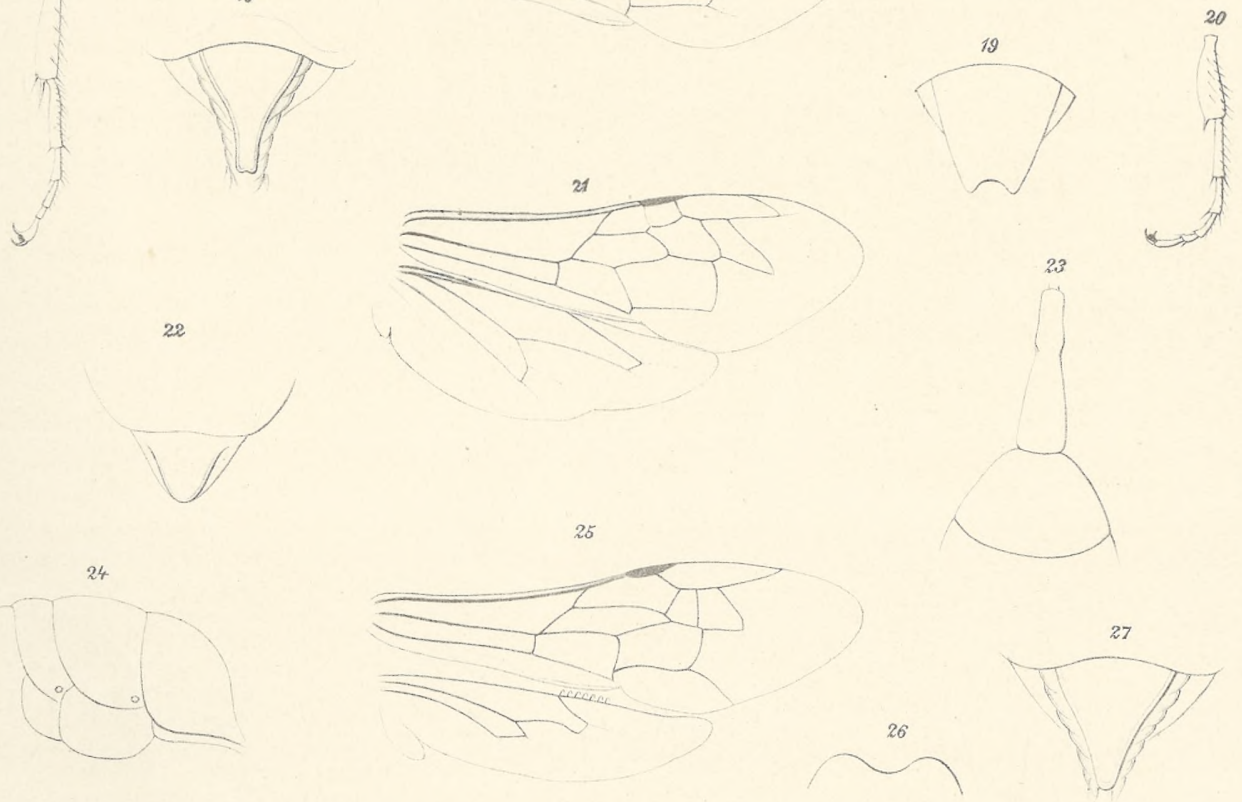

E. Konopicky lith

Annal.d.k.k.Nahurhist.Hofmuseums Band I. 1889 


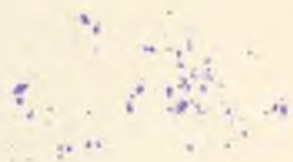

